\title{
ON A GENERAL CLASS OF LINEAR HOMOGENEOUS DIFFERENTIAL EQUATIONS OF INFINITE ORDER WITH CONSTANT COEFFICIENTS*
}

BY

J. F. RITT

\section{INTRODUCTION}

Pincherle, in his classic writings on distributive operations, $\nmid$ has shown that the inversion of distributive operations can be made to depend on the solution of linear differential equations of infinite order. The same result was reached by Bourlet, $\ddagger$ who furthermore undertook the study of such differential equations, but in spite of the title of his memoir, no results appear which would mark a genuine departure from equations of finite order to those whose orders are infinite. In fact, as far as I am aware, no intensive study of such equations has ever been made.

In the present paper such a study is made for the important case where the coefficients are constants, and are subject to one further condition. The first part is given up to the theory of the "entire differential operator of genus zero,"

$$
A=\left(1-\frac{D}{a_{1}}\right)\left(1-\frac{D}{a_{2}}\right) \cdots\left(1-\frac{D}{a_{n}}\right) \cdots,
$$

where $D$ denotes differentiation and where the constants $a_{n}$ are such that

$$
\sum_{n=1}^{\infty} \frac{1}{\left|a_{n}\right|}
$$

is convergent. As far as I know, this operator has never been studied before. Its most notable property is that its domain of applicability consists of all analytic functions. That this property belongs to the linear differential

* Presented to the Society, under a different title, April 29, 1916.

† S. Pincherle, Operazione distributive, p. 136; Mémoire sur le calcul fonctionnel distributif, Mathematische Annalen, vol. 49 (1897), p. 356; Equations et opérations fonctionnelles, Encyclopédie des Sciences Mathématiques, II, 26, p. 25.

‡C. Bourlet, Sur les opérations en général et les équations linéaires différentielles d'ordre infini. Annales de l'école normale, ser. 3, vol. 33 (1897). 
development of $A$ was probably known to Bourlet, although he made no explicit mention of the fact.

The second part contains a discussion of the most general solution of the equation $A \phi(z)=0$. The general properties of the solutions are first obtained-the most striking being, perhaps, that the solutions are all uniformand the analytical representation of the solutions is discussed. In $\S 11$ an application is made to the theory of analytic prolongation, there being obtained, from an entirely new point of view, a known sufficient condition that the circle of convergence of a power series be a natural boundary.

I hope to present later the results of an investigation which I am now conducting on the inversion of other classes of operators.

In notation, I have followed Pincherle, in the main, using capital Roman letters for operators, and small Greek letters for functional symbols. The sum and the product of two distributive operations are defined by the equations

$$
(A+B) \phi(z)=A \phi(z)+B \phi(z), \quad B A \phi(z)=B[A \phi(z)],
$$

respectively. Other questions of notation will be handled as they arise.

Professor Fite has read this paper, as well as the preceding one, and he is responsible for numerous improvements in both. I welcome this opportunity to thank him.

\section{Part 1. The entire differential operator of genus zero}

1. The operator as an infinite product. The reader is familiar with the operator

$$
A_{n}=\left(1-\frac{D}{a_{1}}\right)\left(1-\frac{D}{a_{2}}\right) \cdots\left(1-\frac{D}{a_{n}}\right)
$$

where $a_{1}, a_{2}, \cdots, a_{n}$, are any :eal or complex numbers except zero. We shall call each operator $\left(1-D / a_{n}\right)$ a "factor," and each $a_{n}$ a " zero " of $A_{n}$. The domain of applicability of $A_{n}$ consists of all functions which have $n$ derivatives. The order of the factors of $A_{n}$ is immaterial. It is legitimate to develope $A_{n}$ as a polynomial in $D$ and to apply it as a linear differential operator.

We shall define now the operator

We are to have

$$
A=\left(1-\frac{D}{a_{1}}\right)\left(1-\frac{D}{a_{2}}\right) \cdots\left(1-\frac{D}{a_{n}}\right) \cdots
$$

$$
A \phi(z)=\lim _{n \doteq \infty} A_{n} \phi(z),
$$

so that $A \phi(z)$ will have a meaning provided that $\phi(z)$ has derivatives of all orders, and that the limit involved in the definition exists. Thus, to operate with $A$ will be to operate first with $\left(1-D / a_{1}\right)$, to apply ( $\left.1-D / a_{2}\right)$ 
to the result, etc. When $A \phi(z)$ has a meaning, we may speak of it as being convergent. What we shall mean by the convergence being uniform in a given domain is obvious. We are interested here in the case where

$$
\sum_{n=1}^{\infty} \frac{1}{\left|a_{n}\right|}
$$

is convergent. In that case, we shall call $A$, by reason of an obvious analogy, an " entire differential operator of genus zero." We shall prove, concerning such an operator $A$, the

Theorem I. If $\phi(z)$ is holomorphic in a given domain, $A \phi(z)$ converges in that domain, the convergence being uniform in every closed and bounded domain interior to the given domain.

It follows from a well-known theorem of Weierstrass that $A \phi(z)$ converges to an analytic function.

We shall need the following statement of Taylor's theorem:

Lemma Ia. Denoting by $e^{a D}$ the operator

$$
1+a D+\frac{a^{2} D^{2}}{2 !}+\cdots+\frac{a^{n} D^{n}}{n !}+\cdots,
$$

of which the manner of application is evident, and operating with $e^{a D}$ upon the function $\phi(z)$, analytic for $|z| \leqq r$, where $r>|a|$, we obtain $\phi(z+a)$, which is analytic at least for $|z| \leqq r-|a|$.

We must have also the following lemma:

LEMмA Ib. If $\phi(z)$, analytic for $|z| \leqq r$, has $h$ as the upper bound of its modulus for $|z| \leqq r$, then, if $\left|z_{1}\right|$ and $\left|z_{2}\right|$ are each less than $r-\delta$, where $0<\delta<r$, we have

$$
\left|\phi\left(z_{2}\right)-\phi\left(z_{1}\right)\right|<\frac{h r\left|z_{2}-z_{1}\right|}{\delta^{2}} .
$$

In short, the derivative of $\phi(z)$ will have as a majorant, for $|z| \leqq r$, the function $h r /(r-|z|)^{2}$, so that, for $|z|<r-\delta$,

and

$$
\left|\frac{d \phi(z)}{d z}\right|<\frac{h r}{\delta^{2}}
$$

$$
\left|\phi\left(z_{2}\right)-\phi\left(z_{1}\right)\right|=\left|\int_{z_{1}}^{z_{2}} \frac{d \phi(z)}{d z} d z\right|<\frac{h r\left|z_{2}-z_{1}\right|}{\delta^{2}} .
$$

We shall consider now the convergence of $A \phi(z)$ in the neighborhood of any given point, which point we shall take as the origin for simplicity. Thus, let

$$
\phi(z)=b_{0}+b_{1} z+b_{2} z^{2}+\cdots+b_{n} z^{n}+\cdots,
$$


be regular for $|z| \leqq r$. Now, let

$$
\bar{A}=\left(1+\frac{D}{\left|a_{1}\right|}\right)\left(1+\frac{D}{\left|a_{2}\right|}\right) \cdots\left(1+\frac{D}{\left|a_{n}\right|}\right) \cdots,
$$

and let $A_{n}$ be the operator formed by the first $n$ factors of $\bar{A}$. Also, let

$$
\bar{\phi}(z)=\left|b_{0}\right|+\left|b_{1}\right| z+\left|b_{2}\right| z^{2}+\cdots+\left|b_{n}\right| z^{n}+\cdots .
$$

The majorant $\bar{\phi}(z)$, like $\phi(z)$, is regular for $|z| \leqq r$. Take any $\delta>0$, where $3 \delta<r$. We shall study first the effect of operating on $\bar{\phi}(z)$ with $\bar{A}$, for $|z|<r-3 \delta$. Take any $\epsilon$, positive, but less than $\delta$, and choose an integer $m$ such that, if $n \geqq m$,

$$
\sum_{n+1}^{n+p} \frac{1}{\left|a_{q}\right|}<\epsilon
$$

for every positive integer $p$. The coefficient of any power of $D$ after the first in the development of

$$
\left(1+\frac{D}{\left|a_{n+1}\right|}\right)\left(1+\frac{D}{\left|a_{n+2}\right|}\right) \cdots\left(1+\frac{D}{\left|a_{n+p}\right|}\right)
$$

is less than the coefficient of the same power of $D$ in the development of

$$
e^{\left(\frac{1}{\left|a_{n+1}\right|}+\frac{1}{\left|a_{n+2}\right|}+\cdots+\frac{1}{\left|a_{n+p}\right|}\right) D} .
$$

Hence, for $|z| \leqq r-\delta$ and for $n \geqq m$, we have by Lemma I $a$,

$$
\begin{aligned}
\left(\bar{A}_{n+p}-\bar{A}_{n}\right) \bar{\phi}(|z|) & =\left[\left(1+\frac{D}{\left|a_{n+1}\right|}\right) \cdots\left(1+\frac{D}{\left|a_{n+p}\right|}\right)-1\right] \bar{A}_{n} \bar{\phi}(|z|) \\
& <\bar{A}_{n} \bar{\phi}(|z|+\epsilon)-\bar{A}_{n} \bar{\phi}(|z|) .
\end{aligned}
$$

Let $n$ have the value $m$ for a moment. Since $\bar{A}_{n z} \bar{\phi}(|z|)$ is bounded, for $|z| \leqq r$,

$$
\bar{A}_{m} \bar{\phi}(|z|+\epsilon)-\bar{A}_{m} \bar{\phi}(|z|)
$$

is bounded for $|z| \leqq r-\delta$. Then, by the above inequality, all the functions $\bar{A}_{m+p} \bar{\phi}(|z|)$, or, what is the same, all the functions $\bar{A}_{n} \bar{\phi}(|z|)$, for $n \geqq m$, have a common upper bound $h$ for $|z| \leqq r-\delta .^{*}$ Then, by Lemma $\mathrm{I} b$, we have for $|z| \leqq r-3 \delta$, for any $n$ greater than or equal to $m$, and for any $p$,

$$
\left(\bar{A}_{n+p}-\bar{A}_{n}\right) \bar{\phi}(|z|)<\frac{h(r-\delta) \epsilon}{\delta^{2}}<\frac{h r \epsilon}{\delta^{2}} .
$$

Since the coefficient of any power of $D$ in $\overline{A_{n}}$ is not less than the absolute

* It is essential to bear in mind that although we used an $\epsilon$ in determining $h$, this $h$ depends really only on the sequence of functions $\bar{A}_{n} \bar{\phi}(|z|)$ and can be used again and again while $\epsilon$ is sent to zero by increasing $m$. 
value of the corresponding coefficient in $A_{n}$, it is clear that $\bar{A}_{n} \bar{\phi}(z)$ will be a majorant of $A_{n} \phi(z)$, so that

$$
D^{q} A_{n} \bar{\phi}(|z|) \geqq\left|D^{q} A_{n} \phi(z)\right|,
$$

for $z \leqq r$ and for every $q$. Now we have already seen that

$$
\left(\bar{A}_{n+p}-\bar{A}_{n}\right) \bar{\phi}(|z|)=\left[\left(1+\frac{D}{\left|a_{n+1}\right|}\right) \cdots\left(1+\frac{D}{\left|a_{n+p}\right|}\right)-1\right] \bar{A}_{n} \bar{\phi}(|z|)
$$

and that

$$
\left(A_{n+p}-A_{n}\right) \phi(z)=\left[\left(1-\frac{D}{a_{n+1}}\right) \cdots\left(1-\frac{D}{a_{n+p}}\right)-1\right] A_{n} \phi(z) .
$$

Comparing the corresponding coefficients in the developments of $\bar{A}_{n+p}-\bar{A}_{n}$ and $A_{n+p}-A_{n}$, and taking account of (1), we have

$$
\left|\left(A_{n+\downarrow p}-A_{n}\right) \phi(z)\right| \leqq\left(\bar{A}_{n+p}-\bar{A}_{n}\right) \bar{\phi}(|z|)<\frac{h r \epsilon}{\delta^{2}},
$$

for $|z| \leqq r-3 \delta$ and for $n \geqq m$. Since $h$ and $\delta$ are fixed numbers, and since $\epsilon$ can be made arbitrarily small by a proper choice of $m$, it is clear that $A \phi(z)$ converges uniformly for $|z| \leqq r-3 \delta$. It follows immediately that $A \phi(z)$ converges to a holomorphic function in any domain in which $\phi(z)$ is holomorphic. Also it can easily be shown by means of the Heine-Borel theorem that the convergence is uniform in every closed and bounded domain interior to the domain of regularity of $\phi(z)$. Thus Theorem I is proved.*

It would be natural now to state this theorem for any domain on a Riemann surface. To avoid whatever may be vague in the concept of the most general such surface, we limit ourselves to saying, that if $\phi(z)$ is multiform, $A \phi(z)$ converges uniformly on any curve of finite length along which $\phi(z)$ can be prolonged, whether the curve intersects itself or not. This fact will be very useful to us later.

In the case where $\sum 1 /\left|a_{n}\right|$ is divergent, it is easily seen that $\vec{A} \bar{\phi}(z)$ diverges to $+\infty$ for every positive value of $z$ less than $r$. Thus, the condition that $\sum 1 /\left|a_{n}\right|$ be convergent plays practically the same rôle in the present theory as it does in that of the infinite product.

* Theorem 1 can be extended to the case where each differentiation is preceded by a multiplication by an analytic function $\zeta_{n}(z)$ and is followed by a multiplication by an analytic function $\chi_{n}(z)$, provided all the functions $\zeta_{n}(z), \chi_{n}(z)$ have a common upper bound for their moduli in the given domain. Operators in which each $\zeta_{n}(z)$ is unity and in which $\chi_{n}(z)$ does not vary with $n$, may be reduced to the form of $A$, above, by a suitable change of variable.

Theorem I indicates, and further developments will emphasize, the analogy between the theory of the operator $A$, and that of the ordinary infinite product. One distinction will, however, arise. We shall see, in fact, that although an absolutely convergent infinite product cannot vanish unless one of its factors does, $A \phi(z)$ may very well converge to zero without any $A_{n} \phi(z)$ being identically zero. 
Throughout the rest of this paper, $A$ will stand for an entire differential operator of genus zero.

2. Degree of convergence.* From the inequality (2) above, we infer, since $h, r$, and $\delta$ are fixed once for all, and since $\epsilon$ can be taken as $\sum_{n+1}^{\infty} 1 /\left|a_{q}\right|$, the result:

Theorem II. In any closed and bounded domain interior to the domain of regularity of $\phi(z)$, the convergence of $A \phi(z)$ is at least as rapid as that of $\sum 1 /\left|a_{n}\right|$; that is, the ratio of $\left|A \phi(z)-A_{n} \phi(z)\right|$ to $\sum_{n+1}^{\infty} 1 /\left|a_{q}\right|$ is ultimately less than some finite number.

This is evident for a sufficiently small neighborhood of any point, and the extension to the larger domain is immediate.

An interesting special case presents itself when $A \phi(z)$ converges to zero for all values of $z . \dagger$ Suppose $\phi(z)$ regular for $|z| \leqq r$. Preassigning some positive integer $m$, take $\delta>0$ such that $2 m \delta<r$, and so choose $s$ that, for $n>s$,

$$
\sum_{n+1}^{\infty} \frac{1}{\left|a_{q}\right|}<\delta
$$

Let $\epsilon_{n}$ be the maximum of $\left|A_{n} \phi(z)\right|$ for $|z|=r$. Then a majorant of $A_{n} \phi(z)$ will be $r \epsilon_{n} /(r-|z|)$. Hence, by what we have seen in the proof of Theorem I, we must have, for $|z| \leqq r-2 \delta$, and for $n>s$,

$$
\begin{aligned}
\left|A_{n+p} \phi(z)-A_{n} \phi(z)\right| & \leqq\left[\left(1+\frac{D}{\left|a_{n+1}\right|}\right) \cdots\left(1+\frac{D}{\left|a_{n+p}\right|}\right)-1\right] \frac{r \epsilon_{n}}{r-|z|} \\
& <\frac{r \epsilon_{n} \sum_{n+1}^{\infty} 1 /\left|a_{q}\right|}{\delta^{2}} .
\end{aligned}
$$

Thus, since $A_{n+p} \phi(z)$ approaches zero as $p$ increases,

$$
\left|A_{n} \phi(z)\right| \leqq \frac{r \epsilon_{n} \sum_{n+1}^{\infty} 1 /\left|a_{q}\right|}{\delta^{2}},
$$

for $|z| \leqq r-2 \delta$ and for $n>s$. Carrying out this process $m$ times, with a few slight modifications, we find, finally,

$$
\left|A_{n} \phi(z)\right| \leqq \frac{r^{m} \epsilon_{n}\left(\sum_{n+1}^{\infty} 1 /\left|a_{q}\right|\right)^{m}}{\delta^{2 m}}
$$

for $|z| \leqq r-2 m \delta$. Since, when $\delta$ is once fixed, $\sum_{n+1}^{\infty} 1 /\left|a_{q}\right|$ becomes infinitesimal compared to it as $n$ increases, we infer from (3) the two theorems which follow.

Theorem III. If $A \phi(z)=0$, the modulus of $A_{n} \phi(z)$, at any point, becomes infinitesimal as $n$ increases, compared to the maximum modulus of

* This section can be omitted in a first reading.

$\dagger$ See the final remarks in the footnote on p. 31. 
$A_{n} \phi(z)$ on ahy circle about that point as center, on and within which $\phi(z)$ is regular.

To a certain extent, this fact is not surprising, for it is well known that, in a closed and bounded domain, the modulus of an analytic function assumes its maximum value on the boundary. It is from the intensity of the phenomenon that the theorem derives its interest.

Theorem IV. If $A \phi(z)=0,\left|A_{n} \phi(z)\right|$ becomes less in absolute value than any preassigned power of $\sum_{n+1}^{\infty} 1 /\left|a_{q}\right|$ as $n$ increases.

This is seen, from (3), for a neighborhood of every point, and can be extended immediately to any closed and bounded domain of regularity.

3. The operator $A$ as a linear differential operator. $A$ can be developed formally into a linear differential operator $(A)$, of infinite order. How $(A)$ is to be applied to an analytic function $\phi(z)$, and what we shall mean by the convergence or uniform convergence of $(A) \phi(z)$ in a given domain, are matters on which it is unnecessary to dwell.

Theorem V. If $\phi(z)$ is holomorphic in a given domain, $(A) \phi(z)$ converges to $A \phi(z)$ in that domain, the convergence being absolute, and uniform in every closed and bounded domain interior to the given domain.

Let $(A)_{n}$ be the operator formed by the first $n+1$ terms of $(A) .^{*}$ Choosing first an $\epsilon>0$, take $m$ such that for $n>m$,

$$
\bar{A}_{n+p} \bar{\phi}(|z|)-\bar{A}_{n} \bar{\phi}(|z|)<\frac{1}{2} \epsilon,
$$

for $|z| \leqq r$, and for every $p$. It is easy to see that

$$
(\bar{A})_{n+q} \bar{\phi}(|z|)-\bar{A}_{n} \bar{\phi}(|z|)<\epsilon,
$$

for $|z| \leqq r$ and for every $q$. In short, as $p$ increases, the first $n+q+1$ coefficients in the development of $\bar{A}_{n+p}$ approach the corresponding coefficients in $(\bar{A})_{n+q}$, and $\bar{A}_{n+p}$ will also have terms of higher order, with positive coefficients. Thus, if there is a $q$ for which (5) is not satisfied, (4) will not hold either, for sufficiently large values of $p$.

Now $(\bar{A})_{n+q}-\bar{A}_{n}$ is the linear differential operator formed by the first $n+q+1$ terms in the development of

$$
\begin{aligned}
\left(1+\frac{D}{\left|a_{1}\right|}\right)\left(1+\frac{D}{\left|a_{2}\right|}\right) \cdots\left(1+\frac{D}{\left|a_{n}\right|}\right)\{ & {\left[\left(1+\frac{D}{\left|a_{n+1}\right|}\right)\right.} \\
& \left.\left.\cdots\left(1+\frac{D}{\left|a_{n+q}\right|}\right) \cdots\right]-1\right\},
\end{aligned}
$$

so that the coefficients in the development of $\left(\bar{A}_{n+q}\right)-\bar{A}_{n}$ are positive, and are each not less than the absolute values of the corresponding coefficients in $(A)_{n+q}-A_{n}$. Hence, referring to (5), we have

$$
\left|(A)_{n+q} \phi(z)-A_{n} \phi(z)\right| \leqq \epsilon,
$$

* Observe that $(A)_{n}$ is not the development of $A_{n}$. Trans. Am. Math. Soc. 3 
for $|z| \leqq r$, for $n>m$ and for every $q$. From this last inequality, the truth of Theorem $\mathrm{V}$ for $|z| \leqq r$ follows without difficulty. The extension to the larger domain is immediate. The absolute convergence follows from the convergence of $(\bar{A}) \bar{\phi}(z)$.

Corollary I. The order of the factors of $A$ is immaterial.

In short, whatever be the order of the factors, the same $(A)$ will result.

Corollary II. $A$ is commutative with any power of $D$.

This is clearly a property of $(A)$, and hence one of $A .^{*}$

That $(A) \phi(z)$ always converges was very probably known to Bourlet, although he failed to state the fact explicitly. $\dagger$ Bourlet would have appealed, for the proof, to a theorem by Poincaré on the coefficients of an entire function. On the other hand, our method of proof has put us in a position to prove

Poincare's Theorem. $\ddagger$ If

$$
\phi(z)=c_{0}+c_{1} z+c_{2} z^{2}+\cdots+c_{n} z^{n}+\cdots
$$

is an entire function of genus zero, and if $a$ is any number whatsoever, then

$$
\lim _{n \doteq \infty} n ! a^{n} c_{n}=0 .
$$

Let us operate on $1 /(1-a z)$ with

$$
c_{0}(A)=c_{0}+c_{1} D+c_{2} D^{2}+\cdots+c_{n} D^{n}+\cdots
$$

at the point $z=0$. We get, since

$$
\begin{gathered}
D^{n}\left(\frac{1}{1-a z}\right)_{z=0}=n ! a^{n}, \\
c_{0}(A)\left(\frac{1}{1-a z}\right)_{z=0}=c_{0}+a c_{1}+2 ! a^{2} c_{2}+\cdots+n ! a^{n} c_{n}+\cdots .
\end{gathered}
$$

Poincaré's theorem follows from the convergence of the series above. This theorem is only a special case of a theorem which Poincaré proved, by an entirely different method, for entire functions of any finite genus. $\S$

The two theorems which follow will be of frequent use.

Theorem VI. Given a domain $d$, a closed and bounded domain $d_{1}$ interior to $d$, and a positive number $\epsilon$, we can find a positive number $h$ such that $|A \phi(z)|$ $<\epsilon$ in $d_{1}$ when $\phi(z)$ is holomorphic, and less in absolute value than $h$, throughout $d$.

If $\phi(z)$ is regular for $|z| \leqq r, D^{n} \phi(z)$ will have as a majorant

$$
n ! h r /(r-|z|)^{n+1},
$$

* Cf. Pincherle, Operazione Distributive, p. 119.

† Bourlet, loc. cit., pp. 159 and 161.

$\ddagger$ In the proof, we assume that $c_{0} \neq 0$, but this is not essential.

§ See Borel, Fonctions Entières, Chapter III. 
so that, if $0<\delta<r$,

$$
\left|D^{n} \phi(z)\right| \leqq \frac{n ! r h}{\delta^{n+1}}
$$

for $|z| \leqq r-\delta$. Then, using the expression for $(A)$ above,

$$
|(A) \phi(z)| \leqq \frac{r h}{\delta}\left(1+\frac{\left|c_{1}\right|}{\delta}+\frac{2 !\left|c_{2}\right|}{\delta^{2}}+\cdots+\frac{n !\left|c_{n}\right|}{\delta^{n}}+\cdots\right) .
$$

Referring to the proof of Poincaré's theorem, we see that the series within the parentheses is convergent, so that $|(A) \phi(z)|$ goes to zero with $h$, for $|z| \leqq r-2 \delta$. This proves the theorem for a neighborhood of any point, and the extension to the domain $d_{1}$ is immediate.

Theorem VII. Given the domains $d$ and $d_{1}$ above, and any two positive numbers, $\epsilon$ and $h$, we can determine a positive number $\eta$ such that, if $\sum 1 /\left|a_{n}\right|<\eta$, we have

$$
|A \phi(z)-\phi(z)|<\epsilon
$$

in $d_{1}$, provided $\phi(z)$ is holomorphic, and less in absolute value than $h$, throughout $d$.

The proof, which is very simple, we indicate briefly. If $\phi(z)$ is regular for $z \leqq r$, it will have, in the neighborhood of the origin, a majorant $\bar{\phi}(z)$, which is less in absolute value than $h$ for $|z| \leqq r$. Then, if $\eta<\delta<r$,

$$
|A \phi(z)-\phi(z)| \leqq\left|\bar{A} \bar{\phi}\left(\left|z_{1}\right|\right)-\bar{\phi}(|z|)\right|<\left(e^{\eta D}-1\right) \bar{\phi}(|z|)<\frac{h r \eta}{\delta^{2}}
$$

for $z \leqq r-\delta$. This inequality leads readily to the theorem.

We shall need later, in considering multiform functions, the following modification of Theorem VII.

Theorem $\mathrm{VII}_{1}$. Given a function $\phi(z)$, a curve of finite length on which, inclusive of the extremities, $\phi(z)$ is analytic, and any $\epsilon>0$, we can find an $\eta>0$, such that, when $\sum 1 /\left|a_{n}\right|<\eta$, we have

$$
|A \phi(z)-\phi(z)|<\epsilon
$$

along the curve in question.

4. Distributivity of the operator $A$. From the fact that every $A_{n}$ is distributive, it follows that $A$ is also distributive; that is, as long as only a finite number of functions are involved. To extend the distributivity of $A$ to the sum of an infinite number of functions, we prove the theorem which follows:

TheOREM VIII. If

$$
\psi(z)=\sum_{n=1}^{\infty} \phi_{n}(z)
$$

is uniformly convergent in a given area, each $\phi_{n}(z)$ being holomorphic in that area, we have, in the given area,

$$
A \psi(z)=\sum_{n=1}^{\infty} A \phi_{n}(z),
$$


and the series in the second member of the last equation is uniformly convergent in every closed and bounded domain interior to the given area.

The proof involves the application of a principle stated by Pincherle for all distributive operations.* We have

$$
A \psi(z)=A \sum_{1}^{n} \phi_{q}(z)+A \sum_{n+1}^{\infty} \phi_{q}(z)=\sum_{1}^{n} A \phi_{q}(z)+A \sum_{n+1}^{\infty} \phi_{q}(z) .
$$

But from the uniform convergence of $\sum \phi_{n}(z)$ and from Theorem VI, we see that

$$
A \sum_{n+1}^{\infty} \phi_{q}(z)
$$

goes to zero uniformly in any closed and bounded domain interior to the given area as $n$ increases indefinitely. The theorem is proved.

\section{Multiplication and factorization of operators.}

ThEOREM IX. If $A$ and $B$ are two entire differential operators of genus zero, their product $B A \dagger$ will also be an entire operator of genus zero, and its factors will be the combined factors of $A$ and of $B$.

Obviously, the theorem will be proved if we can show that

$$
B A \phi(z)=\lim _{n \doteq \infty} B_{n} A_{n} \phi(z)
$$

for every analytic $\phi(z)$. We have, identically,

$$
B A \phi(z)-B_{n} A_{n} \phi(z)=B\left(A-A_{n}\right) \phi(z)+\left(B-B_{n}\right) A_{n} \phi(z) .
$$

Now, in any closed and bounded domain, $\left(A-A_{n}\right) \phi(z)$ goes to zero uniformly as $n$ increases, so that, by Theorem VI, $B\left(A-A_{n}\right) \phi(z)$ approaches zero also. We have

$$
\left(B-B_{n}\right) A_{n} \phi(z)=\left\{\left[\left(1-\frac{D}{b_{n+1}}\right)\left(1-\frac{D}{b_{n+2}}\right) \cdots\right]-1\right\} B_{n} A_{n} \phi(z),
$$

where the significarce of the numbers $b$ is evident. Now, since $B_{n} A_{n} \phi(z)$, as we know beforehand, approaches a limit uniformly as $n$ increases, it must stay bounded, as $n$ increases, in any small domain of regularity of $\phi(z)$. Thus, the conditions of Theorem VII hold, and $\left(B-B_{n}\right) A_{n} \phi(z)$ is seen to approach zero. The theorem is proved.

CoRollary I. The product of any finite number of operators of genus zero can be formed by collecting the factors of the separate operators.

Corollary II. The operators $A$ and $B$, above, are commutative.

Conol.lary III. It is legitimate to group the factors of $A$ in any manner, writing $A$ as the product of a finite or an infinite number of operators, each containing a finite or an infinite number of the factors of $A$.

* M a them at is che Annalen, vol. 49 (1897), p. 349.

† See the remarks on notation on p. 28. Note that we do not refer to the formal product of $A$ and $B$. 
For the case of separation into a finite number of operators, this follows directly from Corollary I. In the case of an infinite number of operators, a few other simple considerations are necessary.

\section{Part 2. The homogeneous equation of genus zero}

6. Comparison with the equation of finite order. The object of the second part of this paper is to discuss the most general analytic function $\phi(z)$ such that

$$
A \phi(z)=0 .
$$

We shall call (1) the " homogeneous equation of genus zero."

If $\phi(z)$ satisfies (1) in an arbitrarily small neighborhood, it will satisfy (1) in its entire domain of existence, for, by Theorem I, $A \phi(z)$ is analytic on every curve along which $\phi(z)$ can be prolonged.

Evidently, the solutions of every equation

$$
A_{n} \phi(z)=0
$$

are solutions of (1). Also, if the zeros of $A$ are

of multiplicities

$$
a_{1}, a_{2}, \cdots, a_{n}, \cdots,
$$

respectively, ${ }^{*}$ we have the

$$
p_{1}, p_{2}, \cdots, p_{n}, \cdots
$$

Theorem X. If the series

$$
\phi(z)=\sum_{n=1}^{\infty} e^{a_{n} z}\left(c_{n, 0}+c_{n, 1} z+\cdots+c_{n, p_{n}-1} z^{p_{n}-1}\right)
$$

is uniformly convergent in some area, it satisfies (1) in that area.

This follows from Theorem VIII, since each of the terms of the series is a solution of (1).

Theorem X indicates that (1) has solutions which satisfy no (2), and that the general solution of (1) contains an infinite number of arbitrary constants. It does not furnish a rigorous proof of either of these facts, for we cannot say, as yet, that all of the parameters in the series above are essential. However, we shall show that (1) has solutions which satisfy no (2), $\dagger$ and indeed, in a way which will reveal a striking difference between the two kinds of equations. The solutions of (2) are all entire functions. We shall show that the solutions of (1) may have singularities in the finite part of the plane. The series

$$
e^{z}+e^{22 z}+\cdots+e^{n 2 z}+\cdots,
$$

* Note that

$$
\sum_{n=1}^{\infty} \frac{p_{n}}{\left|a_{n}\right|}
$$

is convergent.

† Cf. the second paragraph in the footnote on p. 31 . 
which is uniformly convergent in any domain in which the real part of $z$ is less than some negative number, is, in such a domain, a solution of (1) when the zeros of $A$ are $1^{2}, 2^{2}, \cdots, n^{2}, \cdots$. As $z$ increases towards zero on the axis of reals, the terms of the series will each approach unity, and the series will tend towards $+\infty$. Thus, the function defined by the series cannot be regular for $z=0$.

Still, the theorem which follows establishes a close connection between (1) and (2).

Theorem XI. The solutions of $A \phi(z)=0$ and the successive integrals of such solutions, are all uniform functions.

It is a question of showing that every analytic $\phi(z)$ such that

$$
A D^{p} \phi(z)=0,
$$

$p$ being any integer, is uniform. Suppose then that the equation above has a multiform solution $\phi(z)$, and let $c$ be a point at which $\phi(z)$ has more than one value. By the second corollary of Theorem $\mathrm{V}$, we have also

$$
D^{p} A \phi(z)=0 \text {. }
$$

There must exist a curve, beginning and ending at $c$, along which $\phi(z)$ can be prolonged, and which leads from one of the values at $c$ to a second.

In operating upon $\phi(z)$ with $A$, it is permissible, by the third corollary of Theorem IX, to apply first the operator

$$
\left(1-\frac{D}{a_{n+1}}\right)\left(1-\frac{D}{a_{n+2}}\right) \cdots
$$

which we shall denote by $A_{n}^{-1} A$, and to follow with $A_{n}$. Thus, $A_{n}^{-1} A \phi(z)$, since it vanishes when operated upon with $D^{p} A_{n}$, is uniform along the curve described above. As $n$ increases indefinitely, we see, by Theorem $\mathrm{VII}_{1}$, that $A_{n}^{-1} A \phi(z)$ approaches $\phi(z)$ along that curve, so that $\phi(z)$ cannot have two distinct values at $c$. The theorem is proved.

Theorem XII. If a solution of $A \phi(z)=0$ is analytic on the entire circumference of a circle, it is analytic throughout the interior of the circle; in particular, no solution of $A \phi(z)=0$ can have an isolated singularity.*

It will suffice, for the proof, to show that $\phi(z)$ cannot have a Laurent development, at any point, in which negative powers are actually present. Suppose, then, that

$$
\begin{aligned}
\phi(z)=b_{0}+b_{1}\left(z-z_{1}\right)+ & b_{2}\left(z-z_{1}\right)^{2}+\cdots+b_{n}\left(z-z_{1}\right)^{n}+\cdots \\
& +b_{-r}\left(z-z_{1}\right)^{-r}+\cdots+b_{-n}\left(z-z_{1}\right)^{-n}+\cdots,
\end{aligned}
$$

where $b_{-r} \neq 0$, the development being valid in some ring about $z_{1}$. Now, since

$$
(A) \phi(z)=\phi(z)+c_{1} D \phi(z)+\cdots+c_{n} D^{n} \phi(z)+\cdots
$$

* We except the case of an isolated singularity at infinity. 
is uniformly convergent in the ring, it is possible, by Weierstrass's theorem, to get the development of $(A) \phi(z)$ at $z_{1}$ by adding up the developments of its separate terms. It is easily seen that the development of $(A) \phi(z)$ must contain the term $b_{-r}\left(z-z_{1}\right)^{-r}$, so that $(A) \phi(z)$ cannot be identically zero. This proves the theorem.

This theorem is also a direct consequence of Theorem XI; for if the Laurent series above contained negative powers, a sufficient number of integrations would introduce a logarithmic term, and one of the integrals of $\phi(z)$ would be multiform.

7. Determination and identification of the formal development. Judging by Theorem $\mathrm{X}$, one might suspect that every solution of $A \phi(z)=0$ is expressible, in all or in part of its domain of existence, ${ }^{*}$ by a uniformly convergent development

$$
\phi(z)=\sum_{n=1}^{\infty} u_{n}=\sum_{n=1}^{\infty} e^{a_{n} z}\left(c_{n, 0}+c_{n, 1} z+\cdots+c_{n, p_{n}-1} z^{p_{n-1}}\right) .
$$

We shall show how this development can be determined when it exists.

Denote by $\left(1-D / a_{n}\right)^{-r} A$, where $r \leqq p_{n}$, the operator

$$
\left(1-\frac{D}{a_{1}}\right)^{p_{1}}\left(1-\frac{D}{a_{2}}\right)^{p_{2}} \cdots\left(1-\frac{D}{a_{n}}\right)^{p_{n}-r}\left(1-\frac{D}{a_{n+1}}\right)^{p_{n+1}} \cdots
$$

Let $\zeta(z)$ be the entire function which results on substituting $z$ for $D$ in the expression for $A$, and let $\zeta^{(n)}(z)$ be the $n$th derivative of $\zeta(z)$.

Let us operate on both sides of (3) with $\left(1-D / a_{n}\right)^{-1} A$. Clearly, the only contribution from the second member of (3) will be from the $n$th term, and then only from $c_{n, p_{n}-1} z^{\dot{p}_{n}-1} e_{\mathrm{n}}^{a_{n} z}$. We find, by direct calculation,

and

$$
\left(1-\frac{D}{a_{n}}\right)^{p_{n}-1} z^{p_{n}-1} e^{a_{n} z}=(-1)^{p_{n}-1} \frac{\left(p_{n}-1\right) !}{a_{n}^{p_{n}-1}} e^{a_{n} z}
$$

$\left(1-\frac{D}{a_{n}}\right)^{-p_{n}} A e^{a_{n 2}}=e^{a_{n 2}}\left(1-\frac{a_{n}}{a_{1}}\right)^{p_{1}} \cdots\left(1-\frac{a_{n}}{a_{n-1}}\right)^{p_{n-1}}\left(1-\frac{a_{n}}{a_{n+1}}\right)^{p_{n+1}} \cdots$.

The second member of the last equation is readily expressed by means of the $p_{n}$ th derivative of $\zeta(z)$, calculated according to Leibnitz's theorem. We find thus,

Hence

$$
\left(1-\frac{D}{a_{n}}\right)^{-p_{n}} A e^{a_{n} z}=\frac{(-1)^{p_{n}} a_{n}^{p_{n}}}{p_{n} !} \zeta^{\left(p_{n}\right)}\left(a_{n}\right) e^{a_{n} z}
$$

$$
\left(1-\frac{D}{a_{n}}\right)^{-1} A \phi(z)=-\frac{a_{n} \zeta^{\left(p_{n}\right)}\left(a_{n}\right)}{p_{n}} c_{n, p_{n}-1} e^{a_{n} z},
$$

* That is, in a two-dimensional part. 
or

$$
c_{n, p_{n}-1}=-\frac{p_{n} e^{-a_{n} z}}{a_{n} \zeta^{\left(p_{n}\right)}\left(a_{n}\right)}\left(1-\frac{D}{a_{n}}\right)^{-1} A \phi(z)
$$

We find, in a similar manner, for the other coefficients in the $n$th term, the recursion formula

$$
\begin{aligned}
c_{n, p_{n}-r}=(-1)^{r} \frac{p_{n} ! e^{-a_{n} z}}{\left(p_{n}-r\right) ! a_{n}^{r} \zeta^{\left(p_{n}\right)}\left(a_{n}\right)}\left(1-\frac{D}{a_{n}}\right)^{r} A[\phi(z) \\
\left.\quad-e^{a_{n} z}\left(c_{n, p_{n}-r+1} z^{p_{n}-r+1}+\cdots+c_{n, p_{n}-1} z^{p_{n}-1}\right)\right] .
\end{aligned}
$$

Formula (4) shows that if a uniformly convergent development (3) exists, it will be unique. The question arises as to whether (3) represents $\phi(z)$ if it is uniformly convergent in a part of the domain of existence of $\phi(z)$. The reply is affirmative. We shall prove, indeed, with greater generality, the following theorem:

TheOREM XIII. If there exists a number $h$ such that

$$
\left|\sum_{1}^{n} u_{q}\right|<h
$$

for every $n$, in an area contained in the domain of existence of $\phi(z)$, the development (3) converges uniformly to $\phi(z)$ in every closed and bcunded domain $d_{1}$ interior to that area.

One of the points involved in the proof will be of great importance later, and is of interest in itself. We therefore stop to give it as a separate theorem.

TheOREM XIV. The result obtained by operating on a sclution"of $A \phi(z)=0$ with all but a finite number of the factors of $A$, is identical with the result obtained by operating formally on the development (3) of that solution, whether the development is valid or not.

We are to operate on a solution, $\phi(z)$, and on its development (3), with

$$
\left(1-\frac{D}{a_{1}}\right)^{r_{1}} \ldots\left(1-\frac{D}{a_{m}}\right)^{r_{m}}\left(1-\frac{D}{a_{m+1}}\right)^{p_{m+1}} \ldots\left(1-\frac{D}{a_{n}}\right)^{p_{n}} \ldots,
$$

where $r_{i} \leqq p_{i}, i=1,2, \cdots, m$. The contribution from the development (3) will come only from the first $m$ terms, $\sum_{1}^{m} u_{q}$. It suffices then to show that we get identical results when we operate on the solution and on its development with

$$
\left(1-\frac{D}{a_{m+1}}\right)^{p_{m+1}} \cdots\left(1-\frac{D}{a_{n}}\right)^{p_{n}} \ldots \text {; }
$$

the application of the finite number of remaining factors will not disturb the equality.

The finite series $\sum_{1}^{n} u_{q}$ is a solution of $A \phi(z)=0$, and thus admits of development into a series (3), by the method exposed above. Since we know beforehand, from the very form of the solution, that a valid development 
exists, and since we have seen that such a development is unique, the process above will conduct us again to the finite series $\sum_{1}^{m} u_{q}$. The function $\phi(z)$ - $\sum_{1}^{m} u_{q}$ is also a solution of $A \phi(z)=0$, and has a development (3). Since the operations effected in obtaining the development (3) are distributive, and since the first $m$ terms in the developments of $\phi(z)$ and of $\sum_{1}^{m} u_{q}$ coincide, the first $m$ terms in the development of $\phi(z)-\sum_{1}^{n} u_{q}$ must be identically zero. Thus, drawing from (4) the coefficient of $e^{a_{i} z}$ in that development, we get, for $i \leqq m$,

Let

$$
\left(1-\frac{D}{a_{i}}\right)^{-p_{i}} A\left[\phi(z)-\sum_{1}^{m} u_{q}\right]=0 \text {. }
$$

Then

$$
\xi_{m}(z)=\left[\left(1-\frac{D}{a_{m+1}}\right)^{p_{m+1}} \ldots\left(1-\frac{D}{a_{n}}\right)^{p_{n}} \ldots\right]\left[\phi(z)-\sum_{1}^{m} u_{q}\right] .
$$

$$
\begin{aligned}
& \left(1-\frac{D}{a_{2}}\right)^{p_{2}}\left(1-\frac{D}{a_{3}}\right)^{p_{3}} \cdots\left(1-\frac{D}{a_{m}}\right)^{p_{m}} \xi_{m}(z)=0 \text { ， } \\
& \left(1-\frac{D}{a_{1}}\right)^{p_{1}}\left(1-\frac{D}{a_{3}}\right)^{p_{3}} \cdots\left(1-\frac{D}{a_{m}}\right)^{p_{m}} \xi_{m}(z)=0 \text { ， } \\
& \left(1-\frac{D}{a_{1}}\right)^{p_{1}}\left(1-\frac{D}{a_{2}}\right)^{p_{2}} \ldots\left(1-\frac{D}{a_{m-1}}\right)^{p_{m-1}} \xi_{m}(z)=0 \text {. }
\end{aligned}
$$

It is a simple matter to show that the differential equations (5) have no common integral except

$$
\xi_{m}(z)=0,
$$

and this establishes our theorem, in virtue of the opening paragraph of the proof.

Now we return to Theorem XIII. By hypothesis, $\left|\phi(z)-\sum_{1}^{m} u_{q}\right|$ stays bounded, as $m$ increases, in a domain to which $d_{1}$ is interior, and, since

$$
\xi_{m}(z)=0,
$$

we see by Theorem VII that $\phi(z)-\sum_{1}^{m} u_{q}$ approaches zero uniformly in $d_{1}$. Theorem XIII is proved.*

8. Second form of the development. Convergence discussion. To discuss the convergence of the development (3), we shall exhibit $\phi(z)$ in a somewhat different form. Without concerning ourselves with questions of convergence, let us suppose that we have, formally,

$$
\frac{1}{\zeta(z)}=\sum_{n=1}^{\infty}\left[\frac{f_{n, 1}}{1-\frac{z}{a_{n}}}+\frac{f_{n, 2}}{\left(1-\frac{z}{a_{n}}\right)^{2}}+\cdots+\frac{f_{n, p_{n}}}{\left(1-\frac{z}{a_{n}}\right)^{p_{n}}}\right]
$$

* The methods of the two theorems just proved permit us easily to show that all solutions common to $A \phi(z)=0$ and $B \phi(z)=0$ are solutions of $C \phi(z)=0$, where the factors of $C$ are the factors common to $A$ and to $B$. 
or

$$
1=\sum_{n=1}^{\infty}\left[\frac{f_{n, 1} \zeta(z)}{1-\frac{z}{a_{n}}}+\frac{f_{n, 2} \zeta(z)}{\left(1-\frac{z}{a_{n}}\right)^{2}}+\cdots+\frac{f_{n, p_{n}} \zeta(z)}{\left(1-\frac{z}{a_{n}}\right)^{p_{n}}}\right]
$$

This being considered as an identity in $z$, the subsistence of a similar identity in $D$ would lead to a development of $\phi(z)$ in the form

$$
\begin{aligned}
\phi(z)=\sum_{n=1}^{\infty} v_{n}=\sum_{n=1}^{\infty}\left[f_{n, 1}\left(1-\frac{D}{a_{n}}\right)^{-1} A \phi(z)\right. & +\cdots \\
& \left.+f_{n, p_{n}}\left(1-\frac{D}{a_{n}}\right)^{-p_{n}} A \phi(z)\right] .
\end{aligned}
$$

We say, in fact, that the $n$th term $v_{n}$ of (6), taken as a whole, is equivalent to the $n$th term $u_{n}$ of (3). If $a_{n}$ is not a multiple zero of $\zeta(z)$, the proof is almost immediate, and doubtless the general case could be handled by mechanical transformations, but the method which follows, though somewhat indirect, will be less painful.

First we observe, referring to Theorem XIV, that

$$
\left(1-\frac{D}{a_{n}}\right)^{-p_{n}} A\left[\phi(z)-u_{n}\right]=0 \text {. }
$$

We shall prove also that

$$
\left(1-\frac{D}{a_{n}}\right)^{-p_{n}} A\left[\phi(z)-v_{n}\right]=0 .
$$

This will follow as soon as we have shown that

$$
v_{n}=f_{n, 1}\left(1-\frac{D}{a_{n}}\right)^{-1} A v_{n}+\cdots+f_{n, p_{n}}\left(1-\frac{D}{a_{n}}\right)^{-p_{n}} A v_{n} .
$$

We know that (6) becomes a true identity if we employ the operator $A_{m}$ instead of $A$, and the partial fraction development of the reciprocal of the first $m$ factors of $\zeta(z)$ instead of that of $1 / \zeta(z){ }^{*}$ Since

$$
\left(1-\frac{D}{a_{n}}\right)^{p_{n}} v_{n}=0 \text {, }
$$

we get, for sufficiently large values of $m$, an identity for $v_{n}$ consisting of $p_{n}$ terms, as in (9). As $m$ increases indefinitely, we approach the expression for $v_{n}$ in (9). Then

$$
\begin{aligned}
f_{n, 1}\left(1-\frac{D}{a_{n}}\right)^{-1} A\left[\phi(z)-v_{n}\right] & +\cdots \\
& +f_{n, p_{n}}\left(1-\frac{D}{a_{n}}\right)^{-p_{n}} A\left[\phi(z)-v_{n}\right]=0 .
\end{aligned}
$$

From (10) follows the truth of (8), for if the last term of (10) were not identi-

* We understand in this that equal zeros are written separately. 
cally zero, it would be the product of $e^{a_{n} z}$ by a polynomial in $z$, while the terms which precede the last would be products of $e^{a_{n} z}$ by polynomials of lower degree, so that (10) would be impossible.*

From (7) and (8),

But, also,

$$
\left(1-\frac{D}{a_{n}}\right)^{-p_{n}} A\left(u_{n}-v_{n}\right)=0 \text {. }
$$

Let

$$
\left(1-\frac{D}{a_{n}}\right)^{p_{n}}\left(u_{n}-v_{n}\right)=0 .
$$

Then

$$
w=\left[\left(1-\frac{D}{a_{n+r}}\right)^{p_{n+r}}\left(1-\frac{D}{a_{n+r+1}}\right)^{p_{n+r+1}} \cdots\right]\left(u_{n}-v_{n}\right) .
$$

$$
\left(1-\frac{D}{a_{1}}\right)^{p_{1}} \cdots\left(1-\frac{D}{a_{n-1}}\right)^{p_{n-1}}\left(1-\frac{D}{a_{n+1}}\right)^{p_{n+1}} \cdots\left(1-\frac{D}{a_{n+r-1}}\right)^{p_{n+r-1}} w=0
$$

and

$$
\left(1-\frac{D}{a_{n}}\right)^{p_{n}} w=0 .
$$

As in $\S 7$, the last two equations can have no common integral except $w=0$, and, as $r$ increases, $w$ approaches $u_{n}-v_{n}$, so that finally,

as was to be proved.

$$
u_{n}-v_{n}=0 \text {, }
$$

The equivalence of (3) and (6) enables us to state sufficient conditions for the convergence of (3) in the entire domain of existence of $\phi(z)$. We say, in fact, that if

$$
\sum_{n=1}^{\infty}\left(\left|f_{n, 1}\right|+\left|f_{n, 2}\right|+\cdots+\left|f_{n, \rho_{n}}\right|\right)
$$

is convergent, the development (3) converges absolutely and uniformly to $\phi(z)$ in every closed and bounded domain interior to the domain of existence of $\phi(z)$.

For the proof, it evidently suffices to show that being given such a closed and bounded domain, there exists a positive number $h$ such that

$$
\left|\left(1-\frac{D}{a_{n}}\right)^{-r} A \phi(z)\right|<h
$$

in that domain, for every $n$ and for $r \leqq p_{n}$. The existence of such an $h$ can be shown by applying Theorem VII, remembering that $A_{m} \phi(z)$ approaches zero as $m$ increases indefinitely. Indeed, it is seen that $\left(1-D / a_{n}\right)^{-r} A \phi(z)$ approaches zero as $n$ increases.

Thus, in virtue of the result of the preceding paper, we can say: If $\zeta(z)$ has only a finite number of multiple zeros, and if there exists an integer

* Observe that we cannot have $f_{n, p_{n}}=0$. 
$r$ such that, for $n>r$,

$$
\left|\frac{a_{n+1}}{a_{n}}\right|>1+\frac{k}{n}
$$

where $k>2$, the development (3) converges absolutely and uniformly to $\phi(z)$ in every closed and bounded domain interior to the domain of existence of $\phi(z)$.

It is possible, however, to obtain a much weaker condition for the validity of (3). Any solution of $A \phi(z)=0$ is also a solution of $B \phi(\varepsilon)=0$, provided the zeros of $A$ are included among the zeros of $B$. Let $\omega$ be a primitive $l$ th root of unity. Then let $B$ possess, together with every zero $a_{n}$ of $A$, the zeros

$$
\omega a_{n}, \omega^{2} a_{n}, \cdots, \omega^{l-1} a_{n} .
$$

If $A$ has some $\omega^{8} a_{n}$ as a zero, as well as $a_{n}$, the above set of zeros will be repeated in $B$. Fvidently, $B$ as thus determined will be an entire operator of genus zero. We may write it

$$
B=\left(1-\frac{D^{l}}{a_{1}^{l}}\right)^{p_{1}}\left(1-\frac{D^{l}}{a_{2}^{l}}\right)^{p_{2}} \ldots\left(1-\frac{D^{l}}{a_{n}^{l}}\right)^{p_{n}} \ldots .
$$

Let $\eta(z)$ be the entire function which results on substituting $z$ for $D^{l}$ in the expression for $B$. Then, corresponding to the formal development of $1 / \eta(z)$ into partial fractions, ${ }^{*}$ we can get a formal development of $\phi(z)$ similar to (6), the operator $D^{l}$ taking the place of $D$. Without modifying greatly the discussion in connection with (6), we can show that the new development of $\phi(z)$ is equivalent to (3), provided we collect into one term, those terms of (3) which arise from distinct zeros of $A$ whose $l$ th powers are equal. We may thus state the theorem:

THEOREM XV. If the sum of the coefficients in the formal development of $1 / \eta(z)$ is absolutely convergent, the development (3) converges absolutely and uniformly to $\phi(z)$ in every closed and bounded domain interior to the domain of existence of $\phi(z)$, provided we unite those terms of (3) which arise from distinct zeros of $A$ whose lth powers are equal. $\dagger$

Now, if

where $k>0$, we have

$$
\left|\frac{a_{n+1}}{a_{n}}\right|>1+\frac{k}{n}
$$

$$
\left|\frac{a_{n+1}^{l}}{a_{n}^{l}}\right|>1+\frac{l k}{n} \text {. }
$$

Since we can so take $l$ that $l k>2$, we have the theorem:

Theorem XVI. If $A$ has only a finite number of multiple zeros, and if there

* Observe that two distinct zeros of $A$ may lead to equal zeros of $\eta(z)$.

$\dagger$ In proving that the development yields $\phi(z)$ it is necessary to extend Theorem XIII to the case where the terms of $\Sigma u_{n}$ are grouped arbitrarily. This extension, like Theorem XIII itself, follows directly from Theorem XIV. 
exists an integer $r$ such that, for $n>r$,

$$
\left|\frac{a_{n+1}}{a_{n}}\right|>1+\frac{k}{n},
$$

where $k>0$, the development (3) converges absolutely and uniformly to $\phi(z)$ in every closed and bounded domain interior to the domain of existence of $\phi(z) .^{*}$

9. Example of a divergent development. Method of summation. If the moduli of the zeros of $A$ do not increase with sufficient rapidity, the development (3) of a solution of $A \phi(z)=0$ may diverge everywhere, or may converge in only a portion of the domain of existence of the solution. It will suffice to give an example of the latter case.

Consider the series

$$
e^{\left(12-\delta_{1}\right) z}-e^{12 z}+e^{\left(22-\delta_{2}\right) z}-e^{22 z}+\cdots+e^{\left(n^{2}-\delta_{n}\right) z}-e^{n^{2 z}}+\cdots,
$$

where each $\delta_{n}$ is some positive number, less than unity, which will be fixed later. This series is uniformly convergent in any domain in which the real part of $z$ is less than some negative number. In short, $\left|e^{\left(n^{2}-\delta_{n}\right) z}\right|$ and $\left|e^{n^{2} z}\right|$ will each be less than $r^{n^{2}-1}$, where $0<r<1$. Also, the series is divergent when the real part of $z$ is positive, for then $\left|e^{n^{2} z}\right|>1$. The analytic function which is represented, perhaps in part, perhaps in all of its domain of existence, by the series above, is a solution of $A \phi(z)=0$ when $A$ has each number $n^{2}$

* If the condition of this theorem is not satisfied, it may be possible to interpolate new zeros between those of $A$ in such a way that the condition is satisfied by the new operator. In that case also, every solution of $A \varphi(z)=0$ has a valid development.

An interesting consequence of this theorem is that every equation $A \varphi(z)=0$ has solutions which are not entire functions. The real and imaginary parts of $a_{n}$ cannot both stay bounded as $n$ increases. To fix our ideas, suppose that the real parts do not stay bounded, and furthermore suppose that we can select a sequence in which the real parts are positive and increase without limit. Of this sequence we can again select a sequence

$$
a_{i_{1}}, a_{i_{2}}, \cdots a_{i_{n}}, \cdots,
$$

in which the imaginary parts are all of one sign, say non-negative, in which the real part of $a_{i_{n}}$ is greater than $n$, and in which the moduli of the zeros increase so rapidly that any solution of $A \varphi(z)=0$ built on these zeros has a development (3) which is valid in the entire domain of existence of the solution. Then the series

$$
\sum_{n=1}^{\infty} e^{a_{i_{n}} z}
$$

is uniformly convergent in any area in which the real part of $z$ is less than some negative number and in which the imaginary part of $z$ is not negative, and is divergent for $z=0$. The solution defined by this series has $z=0$ as a singular point.

In closing this article, it is deserving of notice that the development (6) can be applied to functions which are not solutions of $A \phi(z)=0$. In fact, it is not difficult to show that if the partial fraction development of $1 / \zeta(z)$ converges absolutely to $1 / \zeta(z)$, the development (6) of any analytic $\phi(z)$ converges absolutely and uniformly to $\phi(z)$ in every closed and bounded domain in which $\phi(z)$ is regular. Of course, the development obtained will not generally be a series of exponentials. 
and $n^{2}-\delta_{n}$ for a zero. Now, our series can be written

$$
\sum_{n=1}^{\infty}\left[e^{\left(n^{2}-\delta_{n}\right) z}-e^{n 2 z}\right]
$$

By taking each $\delta_{n}$ sufficiently small, we can make $\left|e^{\left(n^{2}-\delta_{n}\right) z}-e^{n^{2 z}}\right|$ small at pleasure in any bounded domain; for instance, less than $1 / n^{2}$ for $|z|<n$. We find, in this manner, an entire function which is represented only in a limited domain by its formal development (3).

Still, the development (3) is not devoid of significance, even when divergent. It can be converted into a series which represents $\phi(z)$ in its entire domain of existence.

Let us dispense with the exponents $p_{n}$ and write equal zeros of $A$ separately. Since, by Theorem VII, $A_{n}^{-1} A \phi(z)$ approaches $\phi(z)$ as $n$ increases, it is clear that we have

$$
\begin{aligned}
\phi(z)=A_{1}^{-1} A \phi(z)+\left(A_{2}^{-1} A-\right. & \left.A_{1}^{-1} A\right) \phi(z)+\cdots \\
& +\left(A_{n}^{-1} A-A_{n-1}^{-1} A\right) \phi(z)+\cdots,
\end{aligned}
$$

the convergence being uniform in every closed and bounded domain interior to the domain of existence of $\phi(z)$.

The sum of the first $n$ terms of (11) is $A_{n}^{-1} A \phi(z)$, and must vanish if we operate on it with $A_{n}$. Suppose, for brevity, that $A$ has no multiple zeros. The general case will be easy to handle. Then $A_{n}^{-1} A \phi(z)$ can be written in the form

$$
g_{n, 1} e^{a_{1} z}+g_{n, 2} e^{a_{2} z}+\cdots+g_{n, n} e^{a_{n} z} .
$$

Proceeding as in $\$ 7$, we find

$$
g_{n, r}=\frac{e^{-a_{r^{2}}}\left(1-\frac{D}{a_{r}}\right)^{-1} A \phi(z)}{\left(1-\frac{a_{r}}{a_{1}}\right)\left(1-\frac{a_{r}}{a_{2}}\right) \cdots\left(1-\frac{a_{r}}{a_{r-1}}\right)\left(1-\frac{a_{r}}{a_{r+1}}\right) \cdots\left(1-\frac{a_{r}}{a_{n}}\right)} .
$$

As $n$ increases, $g_{n, r}$ approaches the coefficient of $e^{a_{r} z}$ in (3). Thus, (11) may be regarded as a summation of (3), when the latter does not serve to represent $\phi(z)$. To justify this point of view completely, we observe that (11) can be obtained directly from (3). In short, Theorem XIV shows that we can obtain $A_{n}^{-1} A \phi(z)$ by operating formally with $A_{n}^{-1} A$ on (3).

Theorem XVII. The development (11) converges absolutely at every point at which $\phi(z)$ is regular, and at least with the same rapidity as $\sum 1 /\left|a_{n}\right|$.

This follows easily from the identity

$$
\left(A_{n}^{-1} A-A_{n-1}^{-1} A\right) \phi(z)=\frac{1}{a_{n}} A_{n}^{-1} A \frac{d \phi(z)}{d z} .
$$

10. Origin of a development (3). We have seen that a solution of 
$A \phi(z)=0$ can have only one development (3). The important question arises as to whether two different solutions, with non-overlapping domains of existence, may not lead to the same development (3). While we shall settle this question under restricted conditions, we have thus far been unable either to show that different solutions always lead to different developments, or to produce two solutions with the same development. Let us prove the following:

Theorem XVIII. If $A$ is such that the development (3) of every solution $\phi(z)$ of $A \phi(z)=0$ converges absolutely in the entire domain of existence of $\phi(z)$, for instance, if the condition of Theorem $X V$ is satisfied, a series

$$
\sum_{n=1}^{\infty} c_{n} e^{a_{n} z}
$$

can represent only one solution of $A \phi(z)=0$.

If $z_{1}$ and $z_{3}$ are any two points, a point $z_{2}$ on the straight segment joining them is given by

$$
z_{2}=z_{1}+k\left(z_{3}-z_{1}\right)=(1-k) z_{1}+k z_{3},
$$

where $0<k<1$. Then

$$
\left|e^{a_{n} z_{2}}\right|=\left(\left|e^{a_{n} z_{1}}\right|\right)^{1-k}\left(\left|e^{a} z^{z_{3}}\right|\right)^{k} .
$$

It is clear, thus, that $\left|e^{a_{n} z_{2}}\right|$ lies between $\left|e^{a_{n} z_{1}}\right|$ and $\left|e^{a_{n} z_{3}}\right|$. Then, certainly,

$$
\left|e^{a_{n} z_{2}}\right|<\left|e^{a_{n} z_{1}}\right|+\left|e^{a_{n} z_{3}}\right| \text {. }
$$

One sees immediately that if $\sum c_{n} e^{a_{n} z}$ converges absolutely at $z_{1}$ and $z_{3}$, it will converge absolutely and uniformly on the straight segment joining $z_{1}$ and $z_{3}$. It follows also that if the series converges absolutely in two regions of the plane, it converges uniformly in a parallelogram connecting these two regions. Thus, we can pass by analytic prolongation from one region to the other. Theorem XVIII is proved.

For the general question as to whether a development (3) may correspond to two different functions, it will probably be necessary to discuss completely the domains of convergence and of summability of (3). Such a study would be a natural complement to the present investigation, for although we have been able, as in Theorems XI and XII, to find interesting properties of the solutions of $A \phi(z)=0$ by qualitative methods, the solutions would probably present themselves in practice through their developments (3), and it would then be desirable to have knowledge as to the function or functions defined by a given development.

Given a series (3), its theory could be made to depend on that of the convergence of a series (11), for, as we have already seen, we can obtain (11) 
directly from (3). We would find thus a series (11) which converges absolutely in the entire domain of existence of any function $\phi(z)$ which may give rise to the series (3). Also, it is seen that if the series (11) thus obtained converges uniformly in an area, it converges to a function of which the original series (3) is a formal development; the series (11) must therefore converge absolutely in any area in which it converges uniformly. ${ }^{*}$

We shall probably devote a separate paper to these questions.

11. Application to the theory of analytic prolongation. From Theorems XI and XII, it follows immediately that any function which can be represented in an area in its domain of existence by a uniformly convergent series (3), is uniform and has no isolated singularities. The importance of this fact is revealed when we consider a simple type of series (3). Assuming that the numbers $a_{n}$ are positive integers, ordered according to increasing magnitude, consider the series

and with it, the power series

$$
\sum_{n=1}^{\infty} c_{n} e^{a_{n} z},
$$

$$
\sum_{n=1}^{\infty} c_{n} x^{a_{n}} .
$$

If (13) has a radius of convergence $\rho$, (12) will be convergent when the real part of $z$ is less than $\log \rho$ and will be divergent when the real part of $z$ is greater than $\log \rho$. If (13) can be prolonged beyond its circle of convergence, (12) can also be prolonged into its field of divergence. This will be impossible if the development (3) of every solution of $A \phi(z)=0$ converges in the entire domain of existence of the solution. We have thus the result:

If $\sum 1 /\left|a_{n}\right|$ is convergent, and if there exists an integer $r$ such that, for $n>r$,

$$
\left|\frac{a_{n+1}}{a_{n}}\right|>1+\frac{k}{n},
$$

where $k$ is any positive number, the series

$$
\sum_{n=1}^{\infty} c_{n} x^{a_{n}}
$$

has its circle of convergence as a natural boundary.

If a series (13) existed which could be prolonged beyond its circle of convergence, it could be shown that the function thus obtained would be uniform and would have no isolated singularities. Also, we would be able to sum the series throughout the domain over which it could be prolonged,

\footnotetext{
* Perhaps it is well to add, in this connection, that it can be shown as in Theorem.XIII that the development (3) converges uniformly in any closed and bounded domain interior to an area in which $\left|\Sigma_{1}^{n} u_{q}\right|$ stays bounded as $n$ increases, even should that area be exterior to the domain of existence of the solution which gave rise to the development.
} 
according to the method of $\S 9$. There is, however, no possibility of such prolongation. Indeed, Fabry* has given the following sufficient condition for the circle of convergence to be a natural boundary, which can be shown to be satisfied whenever $\sum 1 /\left|a_{n}\right|$ is convergent:

If it is possible to choose a sequence of subscripts $m$ such that

$$
\lim _{m=\infty} \sqrt[a_{m}]{\left|c_{m}\right|}=\lim . \text { sup. } \sqrt[a_{n}]{\left|c_{n}\right|}
$$

and such that the ratio to $a_{m}$ of the number of terms with exponents between $a_{m}(1-\lambda)$ and $a_{m}(1+\lambda)$, where $\lambda$ is an arbitrarily small positive number, goes to zero as $m$ increases, the circle of convergence of (13) is a natural boundary. Columbia Untversity

* E. Fabry, A ct a M a the matic a, vol. 22 (1898), p. 86. See also G. Faber, Muenchener Berichte, vol. 34 (1904). 